\title{
Performance of Internists and Medicine Specialists in Medicare Quality Metrics: Variation by Specialty and Other Physician Characteristics
}

\author{
Andrew B. Rosenkrantz, MD MPA' ${ }^{7}$ Gregory N. Nicola, MD², and Richard Duszak Jr, MD ${ }^{3}$ \\ 'Department of Radiology, NYU School of Medicine, NYU Langone Medical Center, New York, NY, USA; ${ }^{2}$ Hackensack Radiology Group, \\ Hackensack, NJ, USA; ${ }^{3}$ Department of Radiology and Imaging Sciences, Emory University School of Medicine, Atlanta, GA, USA.
}

KEY WORDS: performance measurement; quality assessment; Medicare; health policy.

J Gen Intern Med 34(1):20-2

DOI: $10.1007 / \mathrm{s} 11606-018-4629-\mathrm{x}$

(c) Society of General Internal Medicine 2018

\section{INTRODUCTION}

As healthcare payments shift from volume- to value-based paradigms, Medicare's Quality Payment Program (QPP) was established to accelerate that transition. ${ }^{1}$ Under QPP, most physicians will be scored and paid via the Merit-Based Incentive Payment System (MIPS). MIPS provides a wide range of quality measures for various medical specialties, and its various reporting options include traditional claims-based as well as registry-based and qualified clinical data registry (QCDR)based reporting mechanisms.

Physicians' performance variation under such measures is currently not well understood. For example, it remains unknown whether available measures favor or disfavor certain specialties in obtaining high scores and therefore positive payment adjustments in MIPS. Understanding how various specialty groups have performed to date could help inform the Centers for Medicare \& Medicaid Services (CMS) and professional societies as they jointly develop and implement meaningful and fair metrics, and physician practices as they seek success in MIPS. We aimed to assess performance variation in CMS quality measures among internists and medicine specialists.

\section{METHODS}

The Physician Compare 2015 Individual Eligible Professional Public Reporting Performance Scores data set provides 2015 performance scores for all Medicare-participating providers. ${ }^{2}$ Crosslinking to the separate Medicare Physician Compare National Downloadable File ${ }^{3}$, national provider identification numbers were used to identify physician characteristics, including self-reported primary specialty. National performance was computed for each measure reported by at least 100 providers. Using a previously reported methodology ${ }^{4}$, normalized z-scores were derived for all internists and medicine specialists and for all reported measures by computing the

Published online August 14, 2018 number of standard deviations from the national performance mean for each measure. Accounting for lower scores representing better performance for "inverse" measures, zscores were then averaged across reported measures to obtain a single summary performance measure for each physician. Performance on the most commonly reported measures was identified; univariable associations with physician characteristics were assessed using analysis of variance.

\section{RESULTS}

Among 28,232 internists and medicine specialists, the six most commonly reported measures (Table 1) were all claims-based (rather than registry- or QCDR-based) and included documentation of care in the medical record (average score 95.5\%), tobacco use screening and cessation intervention (96.9\%), body mass index screening and follow-up plan (70.7\%), pneumonia vaccination status for older adults $(66.1 \%)$, influenza immunization (55.1\%), and colorectal cancer screening $(63.0 \%)$. In the full cohort, average \pm SD z-score was 0.132 \pm 0.747 . Performance improved significantly (Table 2 ) with increasing years in practice (z-score range 0.065 to 0.155 ; $p<0.001$ ) and decreasing group practice size (range -0.022 to $0.259 ; p<0.001)$. Physician performance was highest $(p<0.001)$ in the West $(0.197)$ and lowest in the Midwest (0.051). Specialties with best overall performance, in order, were hospitalists (0.307), allergists $(0.225)$, hospice and palliative care physicians $(0.195)$, pulmonologists $(0.181)$, and general internists $(0.176)$. The specialties with worst performance, in order, were transplant cardiologists $(-0.457)$, preventative medicine physicians $(-0.271)$, cardiac electrophysiologists $(-0.090)$, infectious disease physicians $(-0.087)$, and medical oncologists $(-0.040)$.

\section{DISCUSSION}

Overall, internists and medicine specialists reported a diverse set of quality measures to CMS, with no single measure reported by a majority of physicians. Overall performance across measures was associated with various physician characteristics, including experience, group size, and geography. 
Table 1 Performance of Internal Medicine Specialists in Medicare Quality Measures

\begin{tabular}{|c|c|c|c|}
\hline $\begin{array}{l}\text { Reporting mechanism and performance } \\
\text { measures }\end{array}$ & $n$ & Ave & SD \\
\hline \multicolumn{4}{|l|}{ Claims measures } \\
\hline $\begin{array}{l}\text { Documentation of current medications in the } \\
\text { medical record }\end{array}$ & 14,012 & 95.5 & 12.1 \\
\hline $\begin{array}{l}\text { Preventive care and screening: tobacco use: } \\
\text { screening and cessation intervention }\end{array}$ & 10,709 & 96.9 & 7.4 \\
\hline $\begin{array}{l}\text { Preventive care and screening: body mass } \\
\text { index (BMI) screening and follow-up plan }\end{array}$ & 8753 & 70.7 & 27.3 \\
\hline $\begin{array}{l}\text { Pneumonia vaccination status for older } \\
\text { adults }\end{array}$ & 6633 & 66.1 & 27.8 \\
\hline $\begin{array}{l}\text { Preventive care and screening: influenza } \\
\text { immunization }\end{array}$ & 5982 & 55.1 & 31.8 \\
\hline Colorectal cancer screening & 5776 & 63.0 & 31.6 \\
\hline Breast cancer screening & 4636 & 61.2 & 26.9 \\
\hline $\begin{array}{l}\text { Screening or therapy for osteoporosis for } \\
\text { women aged } 65 \text { years and older }\end{array}$ & 2990 & 56.9 & 26.8 \\
\hline $\begin{array}{l}\text { Preventive care and screening: screening for } \\
\text { high blood pressure and follow-up docu- } \\
\text { mented }\end{array}$ & 2451 & 90.0 & 21.0 \\
\hline Diabetes: medical attention for nephropathy & 2120 & 76.3 & 26.0 \\
\hline Pain assessment and follow-up & 1634 & 35.6 & 40.6 \\
\hline Diabetes: foot exam & 1455 & 49.8 & 35.0 \\
\hline Diabetes: eye exam & 1234 & 42.5 & 38.3 \\
\hline Medication reconciliation & 1057 & 96.6 & 10.3 \\
\hline $\begin{array}{l}\text { Elder maltreatment screen and follow-up } \\
\text { plan }\end{array}$ & 741 & 14.0 & 34.5 \\
\hline $\begin{array}{l}\text { Colonoscopy interval for patients with a } \\
\text { history of adenomatous polyps â } € \text { "Avoidance } \\
\text { of Inappropriate Use }\end{array}$ & 465 & 98.0 & 9.1 \\
\hline Falls: risk assessment & 285 & 92.0 & 19.5 \\
\hline $\begin{array}{l}\text { Radiology: exposure time reported for } \\
\text { procedures using fluoroscopy }\end{array}$ & 258 & 98.1 & 8.0 \\
\hline $\begin{array}{l}\text { Chronic obstructive pulmonary disease } \\
\text { (COPD): inhaled bronchodilator therapy }\end{array}$ & 242 & 97.2 & 13.3 \\
\hline Falls: plan of care & 219 & 57.9 & 37.9 \\
\hline $\begin{array}{l}\text { Prevention of central venous catheter } \\
\text { (CVC)-related bloodstream infections }\end{array}$ & 185 & 91.8 & 23.9 \\
\hline $\begin{array}{l}\text { Appropriate follow-up interval for normal } \\
\text { colonoscopy in average risk patients }\end{array}$ & 132 & 93.5 & 14.8 \\
\hline $\begin{array}{l}\text { Osteoarthritis (OA): function and pain } \\
\text { assessment }\end{array}$ & 117 & 86.2 & 31.3 \\
\hline QCDR measures & & & \\
\hline $\begin{array}{l}\text { Photodocumentation of the cecum (also } \\
\text { known as cecal intubation rate) all } \\
\text { colonoscopies }\end{array}$ & 1061 & 96.1 & 7.0 \\
\hline $\begin{array}{l}\text { Photodocumentation of the cecum (also } \\
\text { known as cecal intubation rate) screening } \\
\text { colonoscopies }\end{array}$ & 1046 & 97.0 & 6.2 \\
\hline Adequacy of bowel preparation & 1046 & 94.2 & 7.0 \\
\hline $\begin{array}{l}\text { Documentation of history and physical } \\
\text { rate - colonoscopy }\end{array}$ & 1003 & 98.6 & 4.0 \\
\hline $\begin{array}{l}\text { Hypertension (HTN): blood pressure (BP) } \\
\text { management }\end{array}$ & 967 & 85.5 & 9.8 \\
\hline Appropriate indication for colonoscopy & 842 & 88.2 & 11.2 \\
\hline Registry measures & & & \\
\hline Care plan & 5131 & 69.9 & 34.7 \\
\hline $\begin{array}{l}\text { Preventive care and screening: unhealthy } \\
\text { alcohol use â€ "Screening }\end{array}$ & 2678 & 75.5 & 25.3 \\
\hline Use of high-risk medications in the elderly* & 2628 & 11.6 & 10.9 \\
\hline $\begin{array}{l}\text { Adult kidney disease: blood pressure } \\
\text { management }\end{array}$ & 1393 & 62.3 & 28.0 \\
\hline $\begin{array}{l}\text { Urinary incontinence: assessment of } \\
\text { presence or absence of urinary incontinence } \\
\text { in women aged } 65 \text { years and older }\end{array}$ & 643 & 53.6 & 36.0 \\
\hline $\begin{array}{l}\text { Preventive care and screening: screening for } \\
\text { clinical depression and follow-up plan }\end{array}$ & 518 & 41.5 & 36.0 \\
\hline $\begin{array}{l}\text { Adult kidney disease: laboratory testing } \\
\text { (lipid profile) }\end{array}$ & 377 & 54.7 & 34.1 \\
\hline $\begin{array}{l}\text { Atrial fibrillation and atrial flutter: chronic } \\
\text { anticoagulation therapy }\end{array}$ & 326 & 57.6 & 36.3 \\
\hline $\begin{array}{l}\text { Stroke and stroke rehabilitation: discharged } \\
\text { on antithrombotic therapy }\end{array}$ & 253 & 60.1 & 36.0 \\
\hline
\end{tabular}

Table 1. (continued)

\begin{tabular}{llll}
\hline \hline $\begin{array}{l}\text { Reporting mechanism and performance } \\
\text { measures }\end{array}$ & $n$ & Ave & SD \\
\hline $\begin{array}{l}\text { Diabetes mellitus: diabetic foot and ankle } \\
\text { care, peripheral neuropathy â€'Neurological } \\
\text { Evaluation }\end{array}$ & 239 & 35.2 & 32.7 \\
$\begin{array}{l}\text { Chronic obstructive pulmonary disease } \\
\text { (COPD): spirometry evaluation }\end{array}$ & 182 & 75.5 & 26.7 \\
$\begin{array}{l}\text { Screening colonoscopy adenoma detection } \\
\text { rate measure }\end{array}$ & 155 & 48.9 & 22.6 \\
\hline
\end{tabular}

*Only listed for measures reported by at least 100 internal medicine specialists

Table 2 Overall Performance Among Internal Medicine Specialists, Stratified by Physician Cohorts. Differences Considered Statistically Significant at $p<0.001$

\begin{tabular}{|c|c|c|c|}
\hline$\overline{\text { Cohort }}$ & $\begin{array}{l}\text { Number of } \\
\text { physicians } \\
\text { reporting at } \\
\text { least one } \\
\text { measure with } \\
\text { an associated } \\
\text { z-score }\end{array}$ & $\begin{array}{l}\text { Average } \\
\text { z-score }\end{array}$ & $\begin{array}{l}\text { SD z } \\
\text { score }\end{array}$ \\
\hline \multicolumn{4}{|l|}{ Gender $(p=0.003)$} \\
\hline Female & 7259 & 0.154 & 0.770 \\
\hline \multirow{2}{*}{\multicolumn{4}{|c|}{ Years in practice $(p<0.001)^{*}$}} \\
\hline & & & \\
\hline$<10$ & 3453 & 0.065 & 0.819 \\
\hline $10-24$ & 12,334 & 0.126 & 0.759 \\
\hline $25+$ & 12,246 & 0.155 & 0.712 \\
\hline \multicolumn{4}{|c|}{ Group practice size $(p<0.001)^{*}$} \\
\hline$<10$ & 5535 & 0.259 & 0.696 \\
\hline $10-49$ & 6749 & 0.142 & 0.742 \\
\hline $50-99$ & 2814 & 0.107 & 0.692 \\
\hline $100+$ & 9616 & -0.022 & 0.768 \\
\hline \multicolumn{4}{|c|}{ Geographic region $(p<0.001)^{* *}$} \\
\hline Midwest & 5826 & 0.051 & 0.706 \\
\hline Northeast & 5940 & 0.125 & 0.810 \\
\hline South & 11,705 & 0.148 & 0.751 \\
\hline West & 4727 & 0.197 & 0.695 \\
\hline \multicolumn{4}{|l|}{ Specialty $(p<0.001) * * *$} \\
\hline Hospitalist & 834 & 0.307 & 0.744 \\
\hline Allergy/immunology & 503 & 0.225 & 0.716 \\
\hline Hospice/palliative care & 74 & 0.195 & 0.641 \\
\hline Pulmonary disease & 1502 & 0.181 & 0.677 \\
\hline Internal medicine & 12,718 & 0.176 & 0.792 \\
\hline Gastroenterology & 3367 & 0.158 & 0.680 \\
\hline Hematology/oncology & 946 & 0.153 & 0.703 \\
\hline Critical care (intensivists) & 371 & 0.128 & 0.816 \\
\hline Nephrology & 1172 & 0.101 & 0.697 \\
\hline Hematology & 55 & 0.099 & 0.671 \\
\hline Addiction medicine & 11 & 0.076 & 0.964 \\
\hline Endocrinology & 706 & 0.044 & 0.725 \\
\hline $\begin{array}{l}\text { Cardiovascular disease } \\
\text { (cardiology) }\end{array}$ & 3371 & 0.022 & 0.665 \\
\hline Sleep medicine & 51 & 0.015 & 0.962 \\
\hline Rheumatology & 626 & -0.014 & 0.693 \\
\hline Interventional cardiology & 674 & -0.019 & 0.617 \\
\hline Geriatric medicine & 131 & -0.021 & 0.793 \\
\hline Medical oncology & 245 & -0.040 & 0.766 \\
\hline Infectious disease & 467 & -0.087 & 0.886 \\
\hline Cardiac electrophysiology & 361 & -0.090 & 0.702 \\
\hline Preventative medicine & 22 & -0.271 & 1.308 \\
\hline $\begin{array}{l}\text { Advanced heart failure and } \\
\text { transplant cardiology }\end{array}$ & 24 & -0.457 & 0.568 \\
\hline
\end{tabular}

*Characteristic not known for all physicians

**Excluded when outside of the 50 states or Washington DC

***Specialties listed in descending order of overall performance 
Of note, performance varied widely among specialties, aligning with recent criticism of MIPS by the highly influential Medicare Payment Advisory Commission ${ }^{5}$ and suggesting that any fair comparison of disparate specialties inside the QPP will be difficult. Medicine practices should be aware of such variation in selecting measures to report to MIPS.

Policymakers and national specialty societies should continue to develop comprehensive measure sets, encompassing measures of relevance to all Medicare-participating specialties. As current measures may advantage or disadvantage physicians purely on the basis of their specialty, QPP normalization calibration may be necessary to create a level programmatic playing field.

Corresponding Author: Andrew B. Rosenkrantz, MD MPA; Department of Radiology, NYU School of Medicine, NYU Langone Medical Center, New York, NY, USA (e-mail: Andrew.Rosenkrantz@nyumc.org).

Funding Information Drs. Rosenkrantz and Duszak are supported by research grants from the Harvey L. Neiman Health Policy Institute.

\section{Compliance with Ethical Standards:}

Conflict of Interest: The authors declare that they do not have a conflict of interest.

\section{REFERENCES}

1. Centers for Medicare \& Medicaid Services. Quality Payment Program. https://qpp.cms.gov/. Accessed on July 18, 2018.

2. Centers for Medicare \& Medicaid Serivces. Physician Compare 2015 Individual EP public reporting. https://data.medicare.gov/PhysicianCompare/Physician-Compare-2016-Individual-EP-Public-Report/5qwkyzai/data. Accessed on July 18, 2018.

3. Centers for Medicare \& Medicaid Services. Physician Compare National Downloadable File. https://data.medicare.gov/Physician-Compare/Physician-Compare-National-Downloadable-File/mj5m-pzi6. Accessed on July $18,2018$.

4. Rosenkrantz AB, Niocla GN, Duszak R Jr. Characteristics of highperforming radiologists within Medicare quality programs. Journal of the American College of Radiology. 2018;15(6):842-849.

5. Medicare Payment Advisory Commission. Report to the Congressl Medicare Payment Policy. Chapter 15. March 2018. http://www.medpac.gov/ docs/default-source/reports /mar18_medpac_entirereport_sec.pdf? Accessd on July 18, 2018. 